\title{
Identification and Characterization of PLATZ Transcription Factors in Wheat
}

\author{
Yuxin $\mathrm{Fu}{ }^{1,2}{ }^{-}$, Mengping Cheng ${ }^{1,3}$, Maolian $\mathrm{Li}^{1,3}{ }^{,}$, Xiaojiang Guo ${ }^{1}$, Yongrui $\mathrm{Wu}^{2}$ \\ and Jirui Wang 1,3,4,*(D) \\ 1 Triticeae Research Institute, Sichuan Agricultural University, Chengdu 611130, China; \\ yuxinfu_anna@163.com (Y.F.); 71250@sicau.edu.cn (M.C.); 71296@sicau.edu.cn (M.L.); \\ xiaojiang9945@gmail.com (X.G.) \\ 2 National Key Laboratory of Plant Molecular Genetics, CAS Center for Excellence in Molecular Plant Sciences, \\ Institute of Plant Physiology and Ecology, Shanghai Institutes for Biological Sciences, \\ Chinese Academy of Sciences, Shanghai 200032, China; yrwu@sibs.ac.cn \\ 3 Key Laboratory for Crop Genetic Resources and Improvement in Southwest China, \\ Sichuan Agricultural University, Ministry of Education, Chengdu 611130, China \\ 4 State Key Laboratory of Crop Gene Exploration and Use in Southwest China, \\ Sichuan Agricultural University, Chengdu 611130, China \\ * Correspondence: wangjirui@gmail.com
}

Received: 2 November 2020; Accepted: 18 November 2020; Published: 25 November 2020

check for updates

\begin{abstract}
The PLATZ (plant AT-rich protein and zinc-binding protein) transcription factor family is a class of plant-specific zinc-dependent DNA-binding proteins. PLATZ has essential roles in seed endosperm development, as well as promoting cell proliferation duration in the earlier stages of the crops. In the present study, 62 TaPLATZ genes were identified from the wheat genome, and they were unequally distributed on 15 chromosomes. According to the phylogenetic analysis, 62 TaPLATZ genes were classified into six groups, including two groups that were unique in wheat. Members in the same groups shared similar exon-intron structures. The polyploidization, together with genome duplication of wheat, plays a crucial role in the expansion of the TaPLATZs family. Transcriptome data indicated a distinct divergence expression pattern of TaPLATZ genes that could be clustered into four modules. The TaPLATZs in Module b possessed a seed-specific expression pattern and displayed obvious high expression in the earlier development stage of seeds. Subcellular localization data of TaPLATZs suggesting that they likely perform a function as a conventional transcription factor. This study provides insight into understanding the structure divergence, evolutionary features, expression profiles, and potential function of PLATZ in wheat.
\end{abstract}

Keywords: wheat; transcription factor; PLATZ; seed-specific expression; duplication

\section{Introduction}

Transcription regulation of genes in response to developmental and environmental changes, mediated by the DNA-binding transcription factors (TFs), is an important regulatory mechanism in plants [1]. Transcription factors bind to the cis-acting elements upstream of gene promoters to activate or repress gene expression.

During the evolution of transcription factor families, the TFs between plants and animals or yeast do not always correspond. In Arabidopsis, over 5\% of its genome is devoted to encoding more than 1500 transcription factors, approximately 45\% of which are specific to plants [2]. Some classes of transcription factors appear to have specifically evolved in plants, such as the WRKY, NAC, and AP2/EREBP families, which strictly integrate gene regulatory networks in plant growth processes, including metabolism, hormone signaling, pathogen defense and senescence [3-5]. 
The PLATZ (plant AT-rich sequence and zinc-binding) TF family is a class of plant-specific zinc-dependent DNA-binding proteins whose first member PLATZ1 was isolated in pea. The pea PLATZ1 acts as a transcriptional repressor by nonspecifically binding to A/T-rich sequences [6]. Multiple sequence alignments show that PLATZ proteins possess two highly conserved zinc finger motifs comprising several cysteine and histidine residues, with $\mathrm{C}-\mathrm{x}_{2}-\mathrm{H}-\mathrm{x}_{10}-\mathrm{C}-\mathrm{x}_{2}-\mathrm{C}-\mathrm{x}(4-5)-\mathrm{C}-\mathrm{x}_{2}-\mathrm{C}-\mathrm{x}(3-7)-\mathrm{H}-\mathrm{x}_{2}-\mathrm{H}$ at N-terminal region and $\mathrm{C}-\mathrm{x}_{2}-\mathrm{C}-\mathrm{x}\left({ }_{10-11)}-\mathrm{C}-\mathrm{x}_{3}-\mathrm{C}[6]\right.$, both of which have been described as being necessary for zinc-dependent DNA binding.

PLATZ proteins participate in environmental stress responses. The transcription levels of GmPLATZ1(Glycine max PLATZ1) were upregulated by ABA and drought stress in soybean. Germination in GmPLATZ1-overexpression transgenic Arabidopsis was retarded in response to ABA and osmotic stress [7].

The biological functions of PLATZ proteins were not well identified until the maize $F l 3$ gene was cloned from a classic endosperm semi-dominant mutant [8]. Fl3 (ZmPLATZ12) is specifically expressed in maize endosperm starchy cells, encoding a PLATZ protein, Fl3, that is required for transcription of transfer RNAs (tRNAs) and 5S ribosomal RNA (5S rRNA), through interaction with the RNA polymerase III (RNAPIII) subunits RPC53 and TFC1, which participate in the development of endosperm and filling of storage materials in maize seeds [3].

PLATZ proteins are involved in leaf growth and senescence. ORESARA15 (ORE 15) encodes a PLATZ TF from Arabidopsis, promotes leaf growth by enhancing the rate and duration of cell proliferation in the leaf earlier development stage, and functions as a negative regulator of leaf senescence in the later stage by modulating the GRF/GIF regulatory pathway [9].

The PLATZ gene LOC_Os06g45540 from rice was mapped as a major QTL locus (GL6), identified from flanking sequence analysis of Japonica T-DNA insertion mutant $s g 6$ almost simultaneously, which function in regulating grain sizes. GL6 determines grain length and spikelet number by affecting cell proliferation through gene expression regulation via the RNA polymerase III transcription machinery [10]. SG6 determines grain size by interacting with the core cell cycle machinery DP protein and regulating spikelet hull cell division [11].

PLATZ family members have been identified in several plant species, including 13 genes in the Arabidopsis genome, eight genes in Glycine max, nine genes in Gossypium hirsutum, 15 genes in Oryza sativa, and 17 genes in Zea mays. The PLATZ proteins from rice, maize, and Arabidopsis were clustered into five subfamilies based on their amino acid structures [12]. However, the characterization and function of PLATZ in wheat have not yet been elucidated.

Wheat (Triticum aestivum L., BBAADD) is a staple food crop for more than one-third of the global human population and provides approximately $20 \%$ of the calories consumed by humans globally. Wheat is an allohexaploid species originated from two hybridizations: first between Triticum urartu (AA) and Aegilops speltoides (SS), and second between allotetraploid wild emmer wheat [Triticum turgidum ssp., BBAA] and Aegilops tauschii (DD) [13-15]. Recently, high-quality genome sequencing and assembly have been applied to wheat $[16,17]$. The genome sequences serve as references for underpinning genome scans for phenotypic associations and gene cloning from wheat and their wild progenitors for the accelerated development of improved wheat varieties $[18,19]$. The availability of these reference sequences allows the identification of genes within genetic intervals [20].

Recent advances in wheat genomics have presented an opportunity to identify wheat PLATZ family genes in hexaploid wheat. Identification and functional characterization of PLATZs in wheat will provide an avenue for exploring and verifying the conserved and specific mechanism of PLATZ members in plant development. Here, we characterized wheat PLATZs with respect to the following aspects: (1) genome-wide identification of PLATZ genes in wheat; (2) analysis of conserved motifs and cis-elements of PLATZ genes; (3) identification of the chromosomal distribution and the gene duplications; (4) phylogenetic and evolutionary relationship of PLATZ in wheat associate with rice and maize; (5) elucidation of expression profiles of PLATZ genes; and (6) subcellular localization of PLATZ 
genes. The results provide conditions for further study of PLATZ genes concerning flower and fruit growth in wheat.

\section{Results}

\subsection{Identification of the PLATZ Genes in Wheat}

According to the Hidden Markov Model (HMM) profile (PF04640), a total of 50 candidate sequences were found in the IWGSC v1.1 high-confidence (HC) and low-confidence (LC) peptide database using the HMM search program. This dataset was simplified by keeping the first splice variant from each transcript for further analyses. An additional 13 sequences were identified using a BLAST search in the unannotated IWGSC v2.0 genomic database. Subsequently, the PLATZ domain in the identified sequences was confirmed in the Pfam database, SMART database, and NCBI CDD program. By getting rid of sequences with incomplete PLATZ domains, 62 sequences were identified as putative PLATZ family members for further analysis (Table S1). The number of PLATZs in wheat is approximately three times greater than that of Arabidopsis (13 AtPLATZs), maize (17 ZmPLATZs), and rice (15 OsPLATZs) [12,21]. These TaPLATZs have 145 to 275 amino acids, with an average of 221 amino acids. The molecular weights of TaPLATZ were between $16.67 \mathrm{kDa}$ and $29.76 \mathrm{kDa}$. The predicted pI values of TaPLATZ ranged from 5.21 to 9.68. The 62 identified PLATZ genes contain the conserved cysteine and histidine residues enrichment regions: $\mathrm{C}-\mathrm{x} 2-\mathrm{H}-\mathrm{x}(11-12)-\mathrm{C}-\mathrm{x}_{2}-\mathrm{C}-\mathrm{x}(4-8)-\mathrm{C}-\mathrm{x}_{2}-\mathrm{C}-\mathrm{x}(3-4)-\mathrm{H}-\mathrm{x}_{2}-\mathrm{H}$ and $\mathrm{C}-\mathrm{x}_{2}-\mathrm{C}-\mathrm{x}(10-11)-\mathrm{C}-\mathrm{x}_{3}-\mathrm{C}$. The $\mathrm{N}$-domain and $\mathrm{C}$-domain conserved structure of PLATZ proteins are crucial for their zinc-binding ability [6].

\subsection{Phylogenetic Tree and Conserved Motif Characterization of TaPLATZ Genes}

Multiple sequence alignment data of 62 TaPLATZ genes were used to construct a neighbor-joining phylogenetic tree and further explore the similarity and diversity of motif compositions. TaPLATZs were classified into six groups (Groups I-VI) based on the topological structure of the phylogenetic tree (Figure 1A,B). Group VI was the largest one, containing 16 TaPLATZs. Both Groups I and III had 12 TaPLATZs, followed by Group II and V with 9 TaPLATZs. In contrast, Group IV contained the least, with four TaPLATZs (Table S5).

A neighbor-joining phylogenetic tree was carried out based on PLATZs from wheat associated with maize, and rice (Figure 1A). In most groups, gene phylogeny followed species phylogeny [22]. Only wheat PLATZs could be found in groups V and VI. More members of wheat PLATZs could be found than that from maize and rice in groups I, III, and IV. Some TaPLATZ genes were orthologs to ZmPLATZ and OsPLATZ with 100\% bootstrap value, such as TaPLATZ33, TaPLATZ40, TaPLATZ46 orthologs to ZmPLATZ9 and LOC_Os02g09070 (Group III), revealing the sequences conservation during species evolution.

A total of 12 motifs, named motif- 1 to motif-12, were detected using the MEME online program (Table S4). The highly conserved motif distribution in each group ensures the classification of genes accurately and regulation of downstream genes precisely. However, these motifs showed distinct divergence among six groups (Figure 1C). Motif- 2 and motif- 8 matched to the conservation cysteine and histidine residues in the N-terminal of PLATZ protein, which was found in all groups. Motif-5 and motif-4 had the conservation cysteine residues in the C-terminal of PLATZs that were detected in all groups. Motif-7 represents the termination region of PLATZs in all groups, indicating the integrity of most PLATZs. Motif-10 was detected in all groups except Group V. Motif-6 was only detected in Group IV, Group V, and Group VI; and motif-11 was specific to Group II and V. Motif-12, located in the starting position of translation, was only observed in Group I. All TaPLATZs contained PLATZ-conserved domains in the central region (Figure 1D), providing the ability for zinc-dependent DNA binding. 
A

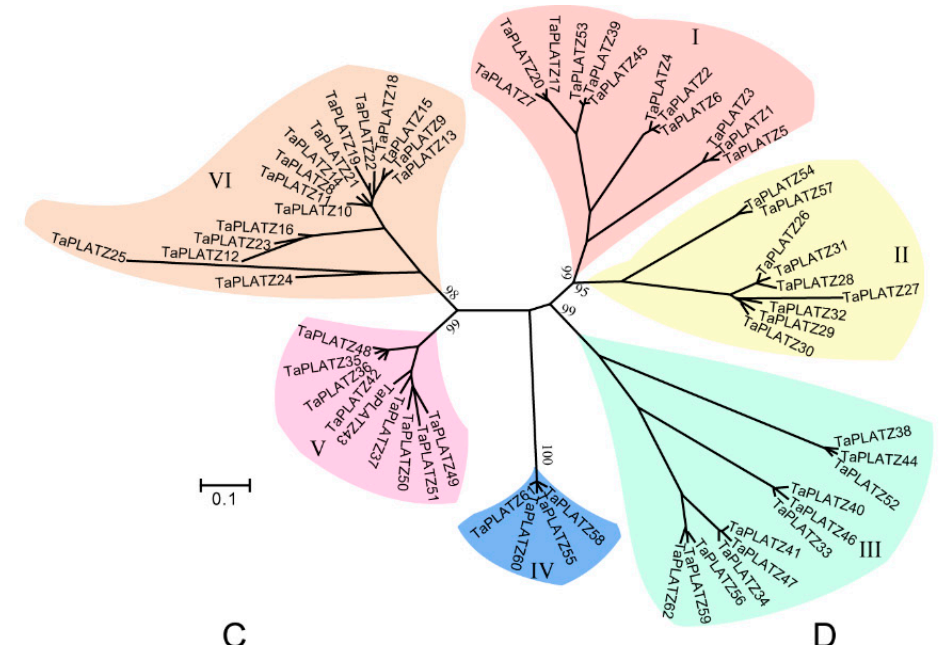

B

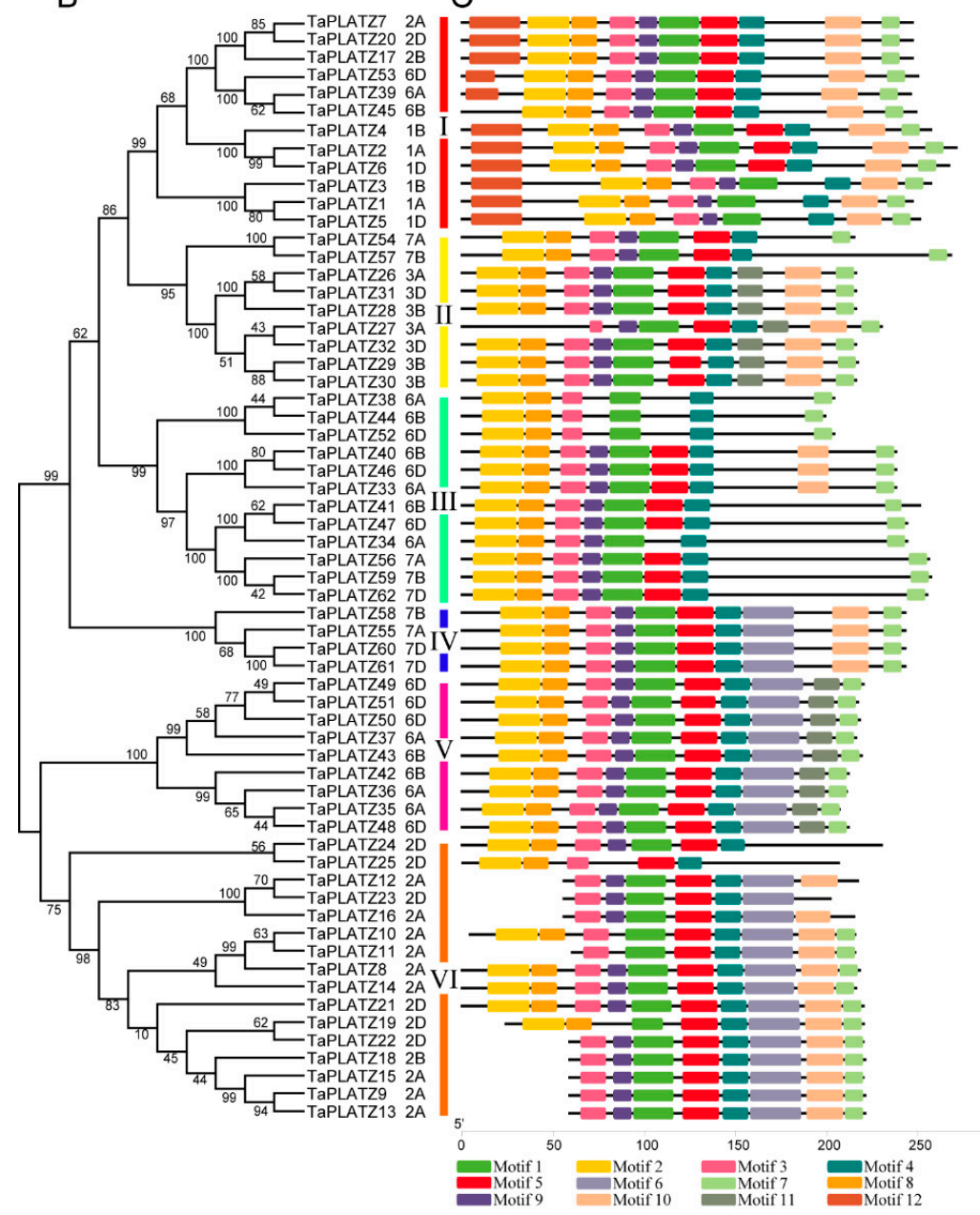

D

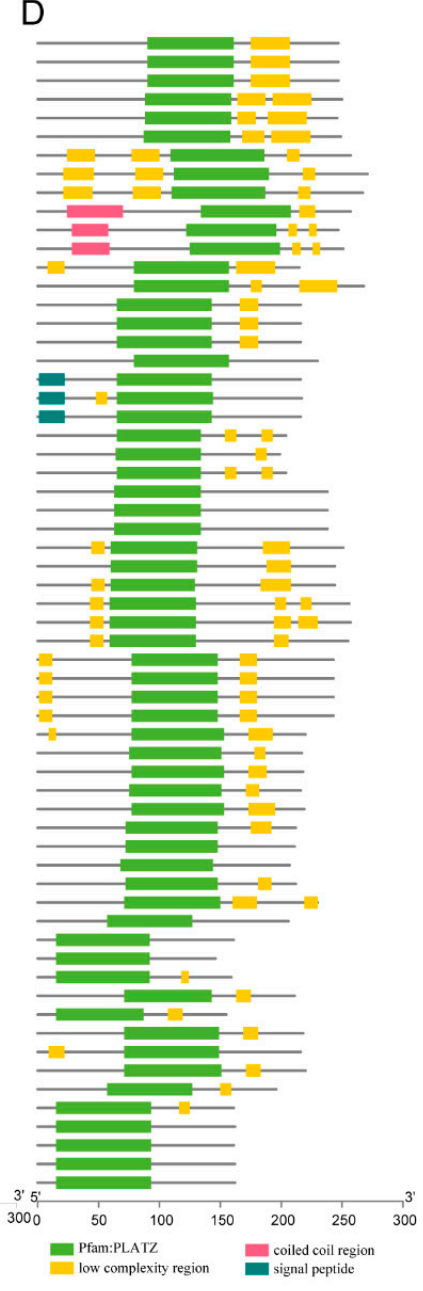

Figure 1. Phylogenetic relationship, motif structure, and conservation analysis of TaPLATZ genes. (A) An unrooted phylogenetic tree of TaPLATZ genes. Percent of bootstrap values are given for the main branches and support the classification in subfamilies; (B) The neighbor-joining tree of TaPLATZ proteins. I-VI: TaPLATZs were divided into six groups and are represented using different colors; (C) MEME motif structure shows the distinct divergence between groups; (D) Batch-smart analysis of PLATZ domain distribution of TaPLATZ proteins. 


\subsection{Collinearity Analysis and Gene Duplication of TaPLATZs}

Collinear relationships between 62 TaPLATZ genes, 15 OsPLATZ genes, and 17 ZmPLATZ genes were analyzed for a better understanding of PLATZ genes evolution (Table S2, Figure 2). A total of thirty TaPLATZ genes showed collinear relationships with those in maize and rice, indicating that these orthologous pairs may already exist before the ancestral divergence. In addition, some TaPLATZ genes were associated with two orthologous genes located on different chromosomes, such as collinear gene pairs (TaPLATZ54, TaPLATZ57, TaPLATZ60, LOC_Os08g44620, and LOC_Os11g24130). Some collinear gene pairs were detected only in wheat and rice, such as TaPLATZ7, TaPLATZ17, TaPLATZ20.

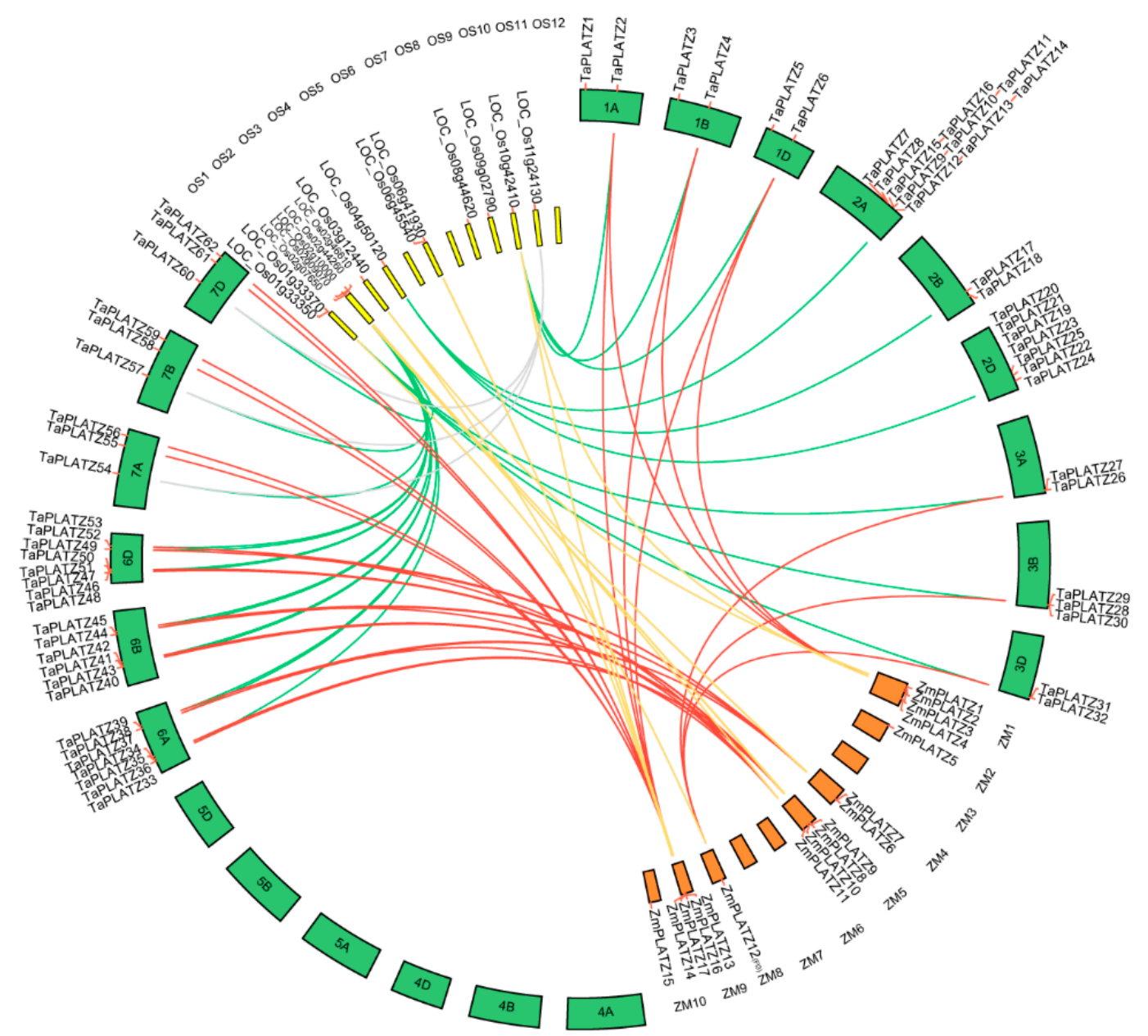

Figure 2. Collinear relationship analysis between orthologous PLATZ genes in wheat, rice, and maize. The circle atlas represents the orthologous pair position and collinearity. The red lines represent the orthologous PLATZ genes between wheat and maize. The green lines denote the orthologous PLAYZ genes in wheat and rice. The yellow lines represent the orthologous genes between maize and rice. Grey lines highlight the second orthologous pair of TaPLATZ54, TaPLATZ57, TaPLATZ60 with rice. 1A-7D represent the twenty-one chromosomes of the wheat; OS1-OS12 represent the twelve chromosomes of rice; ZM1-ZM10 represent the ten chromosomes of maize.

In wheat, all $62 \mathrm{TaPLATZs}$ were found to be unequally distributed on fifteen chromosomes, except for chromosomes 4A, 4B, 4D, 5A, 5B, and 5D. Most of the TaPLATZs (40/62) were located on chromosomes 2A, 2B, 2D, 6A, 6B, and 6D. Meanwhile, chromosomes 1A, 1B, and 1D contained only two TaPLATZs, respectively. It is indicated that duplication events tended to occur in chromosomes 2 and 6 during the evolution of gene families, which may be associated with gene functions. OsPLATZs were distributed unevenly among the nine rice chromosomes, as well. There were no PLATZs on 
chromosomes 5, 7, and 12. In addition, ZmPLATZ also exhibited uneven distribution. None of the PLATZs could be found on chromosomes $3,6,7$, and 10 of maize.

Furthermore, the physical locations of the TaPLATZ genes were mapped on the corresponding chromosomes for a better understanding of the duplication events (Figure 3). During the process of evolution, gene duplication is essential for the generation of novel biological functions and the expansion of the gene family [23]. In wheat, most TaPLATZs had the corresponding homoeologous on the A, B, and D sub-genomes. Forty-six TaPLATZ genes exhibited a homology of 1:1:1 on the three sub-genomes with high identity, and can be referred to as triplets, indicating that wheat polyploidization was the main reason the expansion of the wheat PLATZ family. Apart from triplets, some genes contain several homologues due to complex gene-duplication events during wheat evolution.

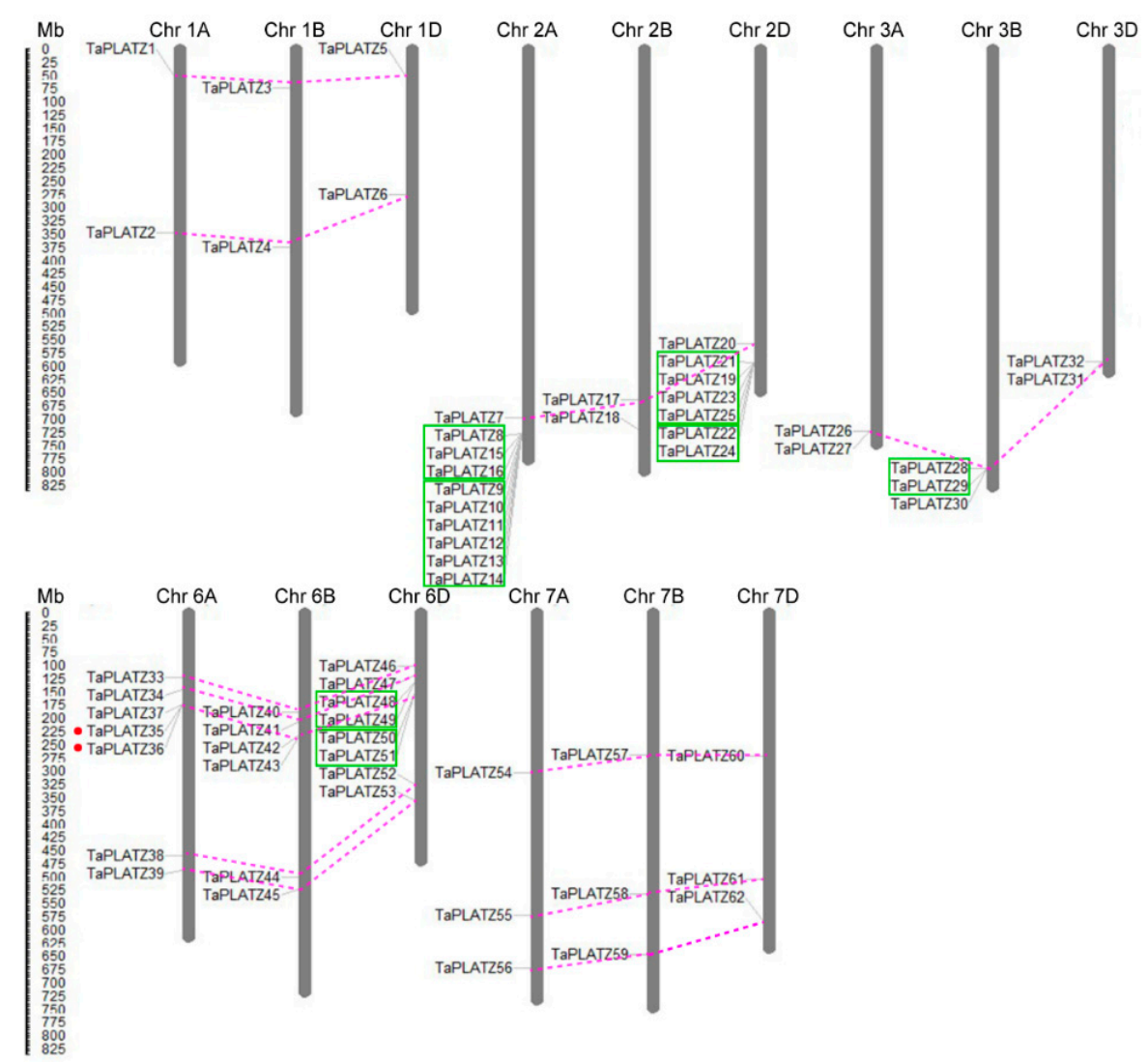

Figure 3. Distribution and duplication of TaPLATZ genes in Triticum aestivum chromosomes. The chromosome numbers are shown at the top of each bar. The length of chromosomes was their relative extent. The scale on the left is in megabases (Mb). Putative TaPLATZ homologous gene pairs were ligated with violet dotted lines. The tandem duplicated genes were marked with green boxes. Segmental duplicated genes were marked by red dots.

Duplication analysis revealed that obvious tandem duplication genes were found universally on chromosome 2A, 2D, 3B, and 6D (Figure 3) according to the criteria used in the analysis. Twenty-one out of $62(33.9 \%)$ TaPLATZ genes were tandem duplicated, based on the similarity of their sequences and the proximity of position on the chromosome (Table S6). In addition, the TaPLATZ family has merely two segmental duplication genes (TaPLATZ35 and TaPLATZ36), indicating that tandem duplication contributed more to the expansion of TaPLATZ family. Moreover, the majority of tandem duplicates were observed on subtelomeric distal regions of chromosomes result in more duplication events. On the other hand, genes located on the centromere proximal region of the chromosomes led to lower exchange frequency, such as TaPLATZs on chromosome 1A, 1B, and 1D. 


\subsection{Variety of Cis-Acting Elements in Promoter Regions of TaPLATZs}

Studies of promoters that largely regulate gene expression at the transcriptional level are crucial for improving our basic understanding of gene regulation [24]. The 2.0-kb upstream sequences of translational initiation sites of TaPLATZs were used to predict cis-acting elements using the online database PlantCARE (Figure 4). Various cis-acting elements were found in the promoter of 62 PLATZ genes. Light responsive elements, including G-box (58/62), Sp1 (35/62), and Box 4 (32/62), were abundant in the 62 TaPLATZ genes. Drought-inducibility element MBS (35/62) accounted for a large part of the TaPLATZ genes, indicating the possibility of binding and regulation by the MYB transcription factor. Hormone responsive elements related to the response of gibberellin, salicylic acid (SA), abscisic acid (ABA), and methyl jasmonic acid (MEJA); as well as various abiotic stresses, such as ABRE (57/62), CGTCA-motif (52/62), and TGACG-motif (45/62), were identified in TaPLATZ genes.

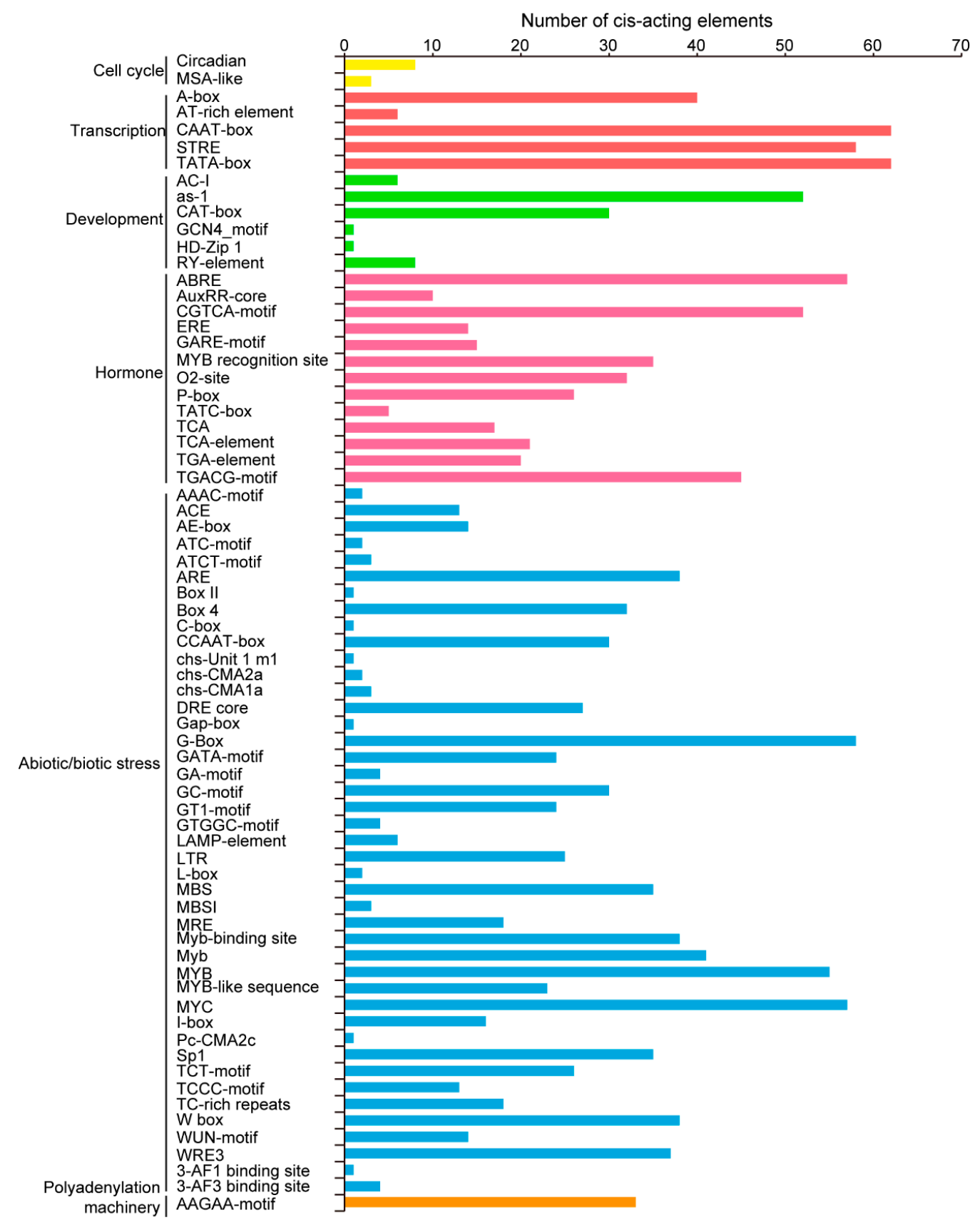

Figure 4. The number of cis-acting elements contained in the promoters of TaPLATZ genes. The cis-acting 
element was identified with the online PlantCARE program using the $2 \mathrm{k}$ upstream from the transcription start site of TaPLATZ genes. The graph was generated based on the presence of cis-acting elements related to different conditions ( $x$-axis) in 62 TaPLATZs ( $y$-axis). The number of cis-acting elements involved in different regulatory pathways. Yellow columns refer to cell cycle, red columns represent transcription, green columns represent of the development process, blue columns refer to abiotic and biotic stress, orange columns represent the polyadenylation machinery.

The promoter-related elements CAAT-box and TATA-box were found in the promoter regions of all the 62 TaPLATZ genes, illustrating that most of the family members have transcriptional activity. Beyond that, eight TaPLATZ genes contained RY-element on promoters, such as TaPLATZ54, TaPLATZ58, and TaPLATZ61, giving rise to the possibility of regulating gene expression during late embryogenesis and seed development stage [25].

\subsection{Expression Patterns of TaPLATZs}

The expression patterns of $62 \mathrm{TaPLATZs}$ were characterized using the transcriptomes (RNA-seq) data at different growth stages or in different tissues of wheat (Figure 5A). TaPLATZs were clustered into four main modules (Module a to $d$ ) in the heat map according to expression abundance. Module $d$ was further divided into four submodules (Module d1 to d4), respectively. Eight TaPLATZs in Module a were highly expressed in roots, stems, leaves, developmental seeds at later stages, which refer to days-post-anthesis (DPA 20, DPA 25, and DPA 30) and germinating seeds after 12 and $24 \mathrm{~h}$ of imbibition (HAI 12, HAI 24). Genes with seed-specific expression patterns were clustered in Module b. Three genes' (TaPLATZ26, TaPLATZ31, TaPLATZ32) high expression levels remained in DPA 5 and DPA 10 seeds, which decreased gradually in DPA 20 and DPA 25. Additionally, the expression level of TaPLATZ30 remained high in all of the developmental stages of the seeds. In Module d1, all genes (TaPLATZ27, TaPLATZ28, and TaPLATZ29) presented seed-specific expression patterns, but maintained a low level of expression. In Module d2, three genes (TaPLATZ6, TaPLATZ2, and TaPLATZ4) were exclusively expressed in roots and leaves. Another three genes clustered in Module d3 (TaPLATZ33, TaPLATZ40, and TaPLATZ46) had an obvious high expression level in roots of SHW-L1 and seeds at HAI 12 and HAI 24 of Chuanmai 32. Two genes (TaPLATZ34, TaPLATZ47) belonging to Module $\mathrm{d} 4$ were only expressed in DPA 30 of Fielder, and seeds at HAI 12, and HAI 24 of Chuanmai 32. In Module c, low expression levels were only detected in a few genes. It is worthy of noting that most genes clustered in the same subfamily of the phylogenetic tree possessed corresponding expression patterns. In other words, homoeologous genes possessed similar expression patterns and further ensured the functional stability in the process of evolution.

Ten TaPLATZs were selected from Module $\mathrm{b}$ and Module $\mathrm{d} 1 \mathrm{and} \mathrm{d} 2$ for real-time PCR, and their expression patterns were further determined in leaves, roots, stems, and different developmental stages of seeds (Figure 5B). The qPCR results of all the selected genes were significantly correlated with the RNA-seq data. The expression levels of three genes (TaPLATZ2, TaPLATZ4, TaPLATZ6) from Module $\mathrm{d} 2$ were relatively high in roots and leaves. Three genes (TaPLATZ27, TaPLATZ28, TaPLATZ29) clustered in Module d1, and four genes (TaPLATZ30, TaPLATZ26, TaPLATZ32, TaPLATZ31) from Module b, presented seed-specific expression patterns. TaPLATZ27 and TaPLATZ28 exhibited the highest expression at DPA 8 , followed by a gradual decrease, subsequently followed by seed development. The expression of TaPLATZ26, TaPLATZ29, TaPLATZ30, TaPLATZ31, and TaPLATZ32 exhibited an up-down-up expression pattern, with a peak at DPA 8 , followed by lower expression at DPA 12 and DPA 16, and then an increase again at DPA 20. 
A

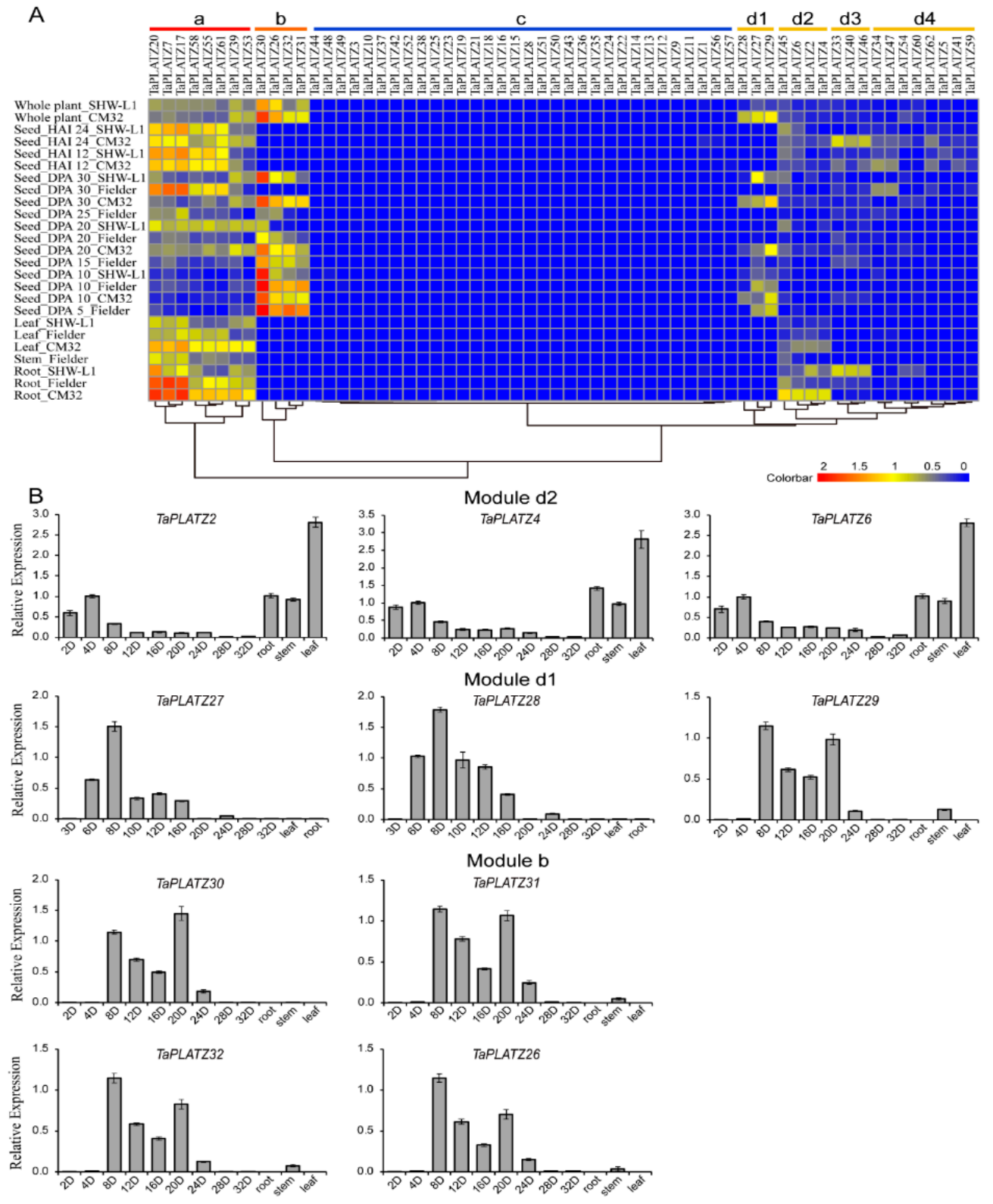

Figure 5. The expression profiles of TaPLATZs in wheat. (A) Heat map of TaPLATZ genes expression in different tissues and developmental stages. Colorbar represents the expression abundance of RNA-seq data. CM32: Chuanmai 32; HAI 12: seeds after $12 \mathrm{~h}$ of imbibition; HAI 24: seeds after $24 \mathrm{~h}$ of imbibition; DPA: days post-anthesis. (B) Real-time PCR data of ten selected TaPLATZs from Modules b, d1, and d2 in different developmental stages of seeds, root, stem, and leaves.

\subsection{Subcellular Localization of TaPLATZ Proteins}

The predicted cellular localization by four different software programs showed that six TaPLATZ proteins (TaPLATZ26, TaPLATZ27, TaPLATZ29, TaPLATZ30, TaPLATZ31, and TaPLATZ32) were located in the nucleus (Table S1). Six TaPLATZ proteins were subsequently chosen to verify the subcellular localization by performing the transient expression. They were fused to a green fluorescent protein (GFP), and the constitutive 355 promoter drove all gene cassettes. The free GFP was used as the control. We transiently expressed the resulting constructs in tobacco leaves. Green fluorescence signals of all fusion proteins were localized in the nucleus (Figure 6), consistent with their predicted function as TFs, whereas the control 35S::GFP was detected both in the nucleus and cytoplasm ubiquitously. 
These results are in accordance with most transcription factors, which were located exclusively at the nucleus, suggesting that the TaPLATZs likely perform a function as a conventional transcription factor.

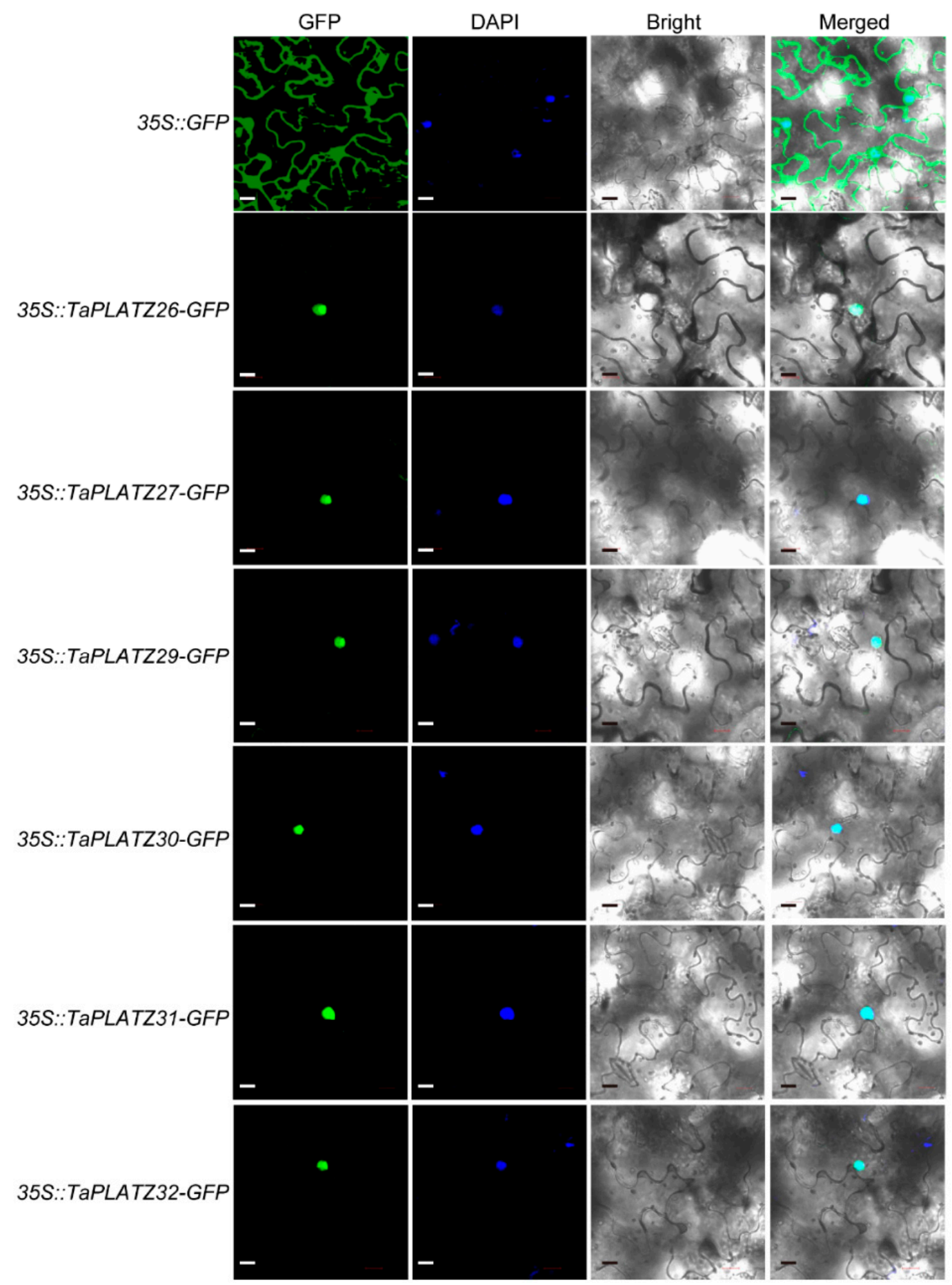

Figure 6. Subcellular localization of TaPLATZ proteins. The localization of the nucleus was detected by DAPI staining. GFP: Green fluorescence indicates the location of TaPLATZ proteins in N. benthamiana. DAPI: Blue fluorescence of DAPI indicated the location of the nucleus. Bright light: field of bright light; Merged: merge with the three former images. The scale bar $=20 \mu \mathrm{m}$.

\section{Discussion}

Ancient duplication events and a high rate of retention of extant pairs of duplicate genes have contributed to an abundance of duplicate genes in plant genomes [26-28]. A total of 62 TaPLATZs 
were characterized in wheat, which was almost threefold the numbers of PLATZs in Arabidopsis (13), rice (15), and maize (17). The phylogenetic tree of PLATZ genes associated with three species revealed that (Figure 1A), the ratio of PLATZ ortholog numbers in wheat to rice/maize was as expected-3:1 (wheat: rice and wheat: maize). However, the ratio within some groups was larger than expected. For example, the ratio was about 7:2 or 7:1 in Group II, and the ratio was 4:1 or 4:1 in Group IV. This large divergence suggests that the PLATZ gene family might have experienced multiple duplication events during the polyploidization of wheat. Gene duplications are considered to be among the primary driving forces in the evolution of genomes and genetic systems [29]. Duplicate genes provide raw materials for the evolution of mechanism novelties, in turn, facilitate the generation of new functions [29]. While in some of the other cases, the ratio was lower than expected. For instance, wheat orthologs of ZmPLATZ2 and ZmPLATZ14 could not be identified in Group III, indicating gene loss during the process of polyploidization of wheat.

Apart from this, among six groups in the phylogenetic tree of three PLATZ families (Figure 1A), genes clustered in Group V and VI have no ortholog gene in neither maize and rice PLATZ family. After sequence alignment, the amino acid sequence of TaPLATZs from Group V and VI showed the highest similarity of TaPLATZs in Group II. The large number of homoeologs can be inferred that most of TaPLATZ genes from Group V and VI may be retained after whole-genome duplications (WGDs).

The wheat (BBAADD) has a complex genome consisting of three related sub-genomes that were derived from three different diploid species [30]. The TaPLATZs were unequally distributed in sub-genomes A, B, and D, which included 24, 16, and 20 members, respectively (Table S1, Figure 2). This suggests that homologous genes on the B genome may be absent or become pseudogenes in the lineage leading to wheat [31]. During the evolutionary process of wheat, rapid alterations and sporadic changes in wheat genome took place due to hybridization, polyploidization, domestication, and mutation, resulting in some modifications and a high level of gene loss [32]. Previous reports have stated that the preferential retention of dosage-sensitive genes (e.g., regulatory genes such as transcription factors) and gene loss following WGDs played a significant role in the evolution of eukaryotes [33].

Through the analysis of the evolutionary mechanism, it has been determined that segmental duplication, tandem duplication, and transposition events, including retro-position and replication transposition, had an essential role in the expansion of the number of genes [34,35]. Among these patterns, segmental and tandem duplications are involved in the main patterns in plant gene family expansion [36]. The occurrence of segmental duplications in plant species was considered to be associated with plant polyploidization, followed by inter-chromosomal rearrangements [35].

Tandem duplications are identified by multiple members in one family occurring within the same intergenic region or in neighboring intergenic regions, results from unequal crossing-over and led to increasing or decreasing copy numbers in gene families [36]. Duplication analysis revealed that twenty-one TaPLATZ genes were identified as tandem duplicates (Figure 2, Table S6), which account for $37.9 \%$ of TaPLATZ duplicates, whereas segmental duplication genes account for $3.2 \%$, indicating that tandem duplication pattern probably played a pivotal role in the expansion of TaPLATZ gene family. TaPLATZ genes are located in the subtelomeric region on chromosome leading to a high percentage of duplication events and results in the expansion of groups. Conversely, genes belonging to groups containing smaller members tended to be located close to the centromere of the chromosomes.

A variety of TaPLATZ gene expression patterns was shown in the heat map (Figure 5A). TaPLATZs genes in Module a displayed a continuous expression except in the early stage of seed development, and TaPLATZs genes in Module b presented a seed-specific expression pattern. Moreover, root-specific expression patterns can be found in Module d, indicating the functionally important and nonredundancy of TaPLATZ genes. TaPLATZ genes clustered in one subfamily could exhibit different expression patterns. For example, members in Group II were clustered into three modules, revealing that functional divergence and the biased expression of duplicated genes appear to be major factors promoting their retention in the genome $[37,38]$. It is noteworthy that the expression patterns of seven TaPLATZs 
(TaPLATZ26 to TaPLATZ32) contained in Group II were highly similar to those of ZmPLATZ12 (Fl3) and LOC_Os01g33350, LOC_Os01g33370, which displayed invariant seed-specific expression pattern in the early stage of seeds. These conserved expression patterns reflect that these TaPLATZs may be involved in seed development and maturation, indicating the possibility of their participation in the common metabolic and or developmental processes of wheat $[8,12]$.

Plants can respond and coordinate growth and stress tolerance to promote survival from abiotic and biotic stresses by modifying the production, distribution, or signal transduction of hormones. Zinc-finger transcription factors are a relatively large family of plant transcription factors (approximately $15 \%$ of the total), which regulate the expression of several genes in response to abiotic stress such as low temperature, salt, drought, osmotic stress, and oxidative stress [2,39]. AtPLATZ1 and AtPLATZ12 were identified as major nodes to positively regulate the acquisition of desiccation tolerance in Arabidopsis seeds and vegetative tissues [40]. The transcription level of GhPLATZ1 (Gossypium hirsutum PLATZ1) was induced by abiotic and hormone stimuli in 20-day-old seedlings. Ectopic expression of GhPLATZ1 in Arabidopsis resulted in enhanced insensitivity to osmotic stresses, ABA, and PAC [41]. Similarly, the RNA expression level of GmPLATZ1 (Glycine max PLATZ1) dramatically increased when responding with exogenous ABA application on soybean plants, the leaves mRNA level of GmPLATZ1 steadily increased after dealing with $24 \mathrm{~h}$ drought stress. Furthermore, ectopic expression of GmPLATZ1 in Arabidopsis showed retarded germination during the early germination process with the addition of mannitol, ABA, and osmotic stress [7]. It is essential to investigate the RNA expression level of TaPLATZs under various abiotic stresses in further study, which will facilitate the identification of potential components to coordinate seedling growth during germination.

\section{Materials and Methods}

\subsection{Plant Growth Conditions}

The wheat landrace Chinese spring was planted in plant growth chambers in a 16-h-light/8-h-dark photoperiod at $20^{\circ} \mathrm{C}$ in 2019 . Tobacco (Nicotiana benthamiana) was grown in the plant growth chamber under a 16-h-light/8-h-dark photoperiod at a temperature of $20 / 25^{\circ} \mathrm{C}$.

\subsection{Identification of TaPLATZ Family Members in the Wheat}

The wheat protein sequence was obtained from the IWGSC database (https://urgi.versailles.inra.fr/ download/iwgsc/IWGSC_RefSeq_Annotations/v1.1/). The Hidden Markov Model (HMM) analysis was carried out for the desired sequences search. The HMM profile of the PLATZ (PF04640) downloaded from the Pfam database (http://pfam.xfam.org/) was applied as a query using HMM search program (http://hmmer.janelia.org/) with an E-value cutoff of 1.0. The protein sequences containing complete or partial PLATZ domain, which may be pseudogenes, incomplete assemblies, sequencing errors, or mispredictions [42], were considered as putative TaPLATZs. To eliminate the TaPLATZ sequences contained incomplete PLATZ domains, a BLASTP program was performed by using identified amino acid sequences as queries with an $e$-value $\leq 1 \times 10^{-3}$. Additionally, the amino acid sequences of maize and rice PLATZ genes were obtained from PlantTFDB (http://plntfdb.bio.uni-potsdam.de/v3.0/) and GrassTFDB (http://www.grassius.org/grasstfdb.php) databases, which were also used for blasting against wheat genomic reference in the IWGSC v2.0 database.

TaPLATZ protein sequences were reconfirmed by Pfam (http://pfam.xfam.org/), SMART (http: //smart.embl-heidelberg.de/), and NCBI-CDD (https://www.ncbi.nlm.nih.gov/cdd/) to identify the conserved PLATZ domain. The sequences lacking the PLATZ domain were excluded. All of the non-redundant and high-confidence genes were named after their chromosomal positions on pseudomolecules. The putative TaPLATZ protein sequences were submitted to CDD (https: //www.ncbi.nlm.nih.gov/Structure/bwrpsb/bwrpsb.cgi). 


\subsection{Characterization of TaPLATZ: Conserved Motif, PLATZ Domain and Putative Cis-Acting Elements}

Conserved motifs of TaPLATZ protein were identified using the MEME website (http://memesuite.org/tools/meme) [43] with the following parameters: distribution of motifs, 0 or 1 occurrence per sequence; maximum number of motifs, 12; minimum sites, 6; maximum width 50 . Visualized of PLATZ domains on each TaPLATZ proteins were performed using TBtoolssoftware [44] (v1.046, Chen, C., GZ, China).

The 2.0-kb upstream of the transcription start site $(-1)$ of TaPLATZs was extracted as a promoter to predict cis-acting elements using the PlantCARE (http://bioinformatics.psb.ugent.be/webtools/ plantcare/html/) [45]. Then, statistics derived from hits of various cis-acting elements were constructed and displayed by the diagram. Theoretical pI/MW of TaPLATZs was calculated by the Compute pI/MW tool (http://web.expasy.org/compute_pi/).

\subsection{Phylogenetic Analysis, Collinear Relationships and Classification of PLATZ Genes in Wheat, Maize and Rice}

The amino acid sequences of PLATZ derived from maize and rice (Table S1), together with newly identified TaPLATZs were used for phylogenetic analysis. All of the amino acid sequences are first aligned by ClustalW with the default parameters. Subsequently, the Bayesian and Neighbor-joining phylogenetic trees were constructed using MEGA software with a bootstrap test of 1000 times [46] (v6.0, Tamura, K., Tokyo, Japan). The TaPLATZs were classified into different groups according to the topology of the phylogenetic tree and the classification in maize and rice from previous studies [12]. The collinear relationships of orthologous PLATZ genes in wheat, maize and rice were displayed using the Circos program [47] (Krzywinski, M., Vancouver, BC, Canada).

\subsection{Location of TaPLATZ Genes on the Chromosome; Identification of Duplication Genes}

The obtained chromosomal location information of TaPLATZ genes was visualized by performing MapInspect software (http://www.softsea.com/download/MapInspect.html) (R. van Berloo, Wageningen, The Netherlands)according to their chromosome locus and the length of each chromosome.

The duplication gene pairs in the TaPLATZ family were identified by BLASTP based on the criteria of the previous studies [48,49]: (a) the alignment covered $>80 \%$ of the longer gene; (b) the aligned region had an identity $>80 \%$.

Tandem duplicated PLATZ genes were defined as two or more adjacent homologous genes located physically on a single chromosome with an intergenic region less than $200 \mathrm{~kb}$ [50], while homologous genes with an interval greater than $200 \mathrm{~kb}$, or between different chromosomes, were defined as segmentally duplicated genes [51].

\subsection{Expression Profiles of TaPLATZ}

A total of 25 RNA-seq (transcript) data files with wheat tissues (root, leaf, stem, grain and spike) at different developmental stages of the hexaploidy bread wheat (Chuanmai 32, SHW-L1 and Fielder) were obtained from the local database, the abbreviation of each tissue corresponding to the detailed description in Table S7.

The transcriptional results were sorted based on the phylogeny classification and visualized in a heatmap using the 'ggplot2' and 'heatmap' R-software (Ihaka R, Auckland, CA, USA)package with a normalization according to an individual gene. The bar graph represents the TaPLATZs expression in tissues. The blocks close to Red representing high expression levels, yellow indicating medium expression levels, and blue refers to almost no expression.

For real-time PCR, tissues including root, stem, the third leaf and seeds were obtained from at least three healthy seedlings and three spikelets after sowing. The roots, stems were isolated from the seedling after one-week after imbibition. The seeds were obtained from spikelets at 2 , $3,6,8,10,12,16,20,24,28$, and 32 days after pollination, respectively. RNA was extracted from 
wheat tissues using the RNA extraction kit (Magen, Beijing, China, Lot: R4165-02), refering to the manufacturer's instruction for specific steps, and then digested with RNase-free DNase I. The quantity and concentration of RNA were evaluated by Thermo Scientific ${ }^{\mathrm{TM}}$ NanoDrop $^{\mathrm{TM}}$. The first-strand cDNA was generated using PrimeScript ${ }^{\mathrm{TM}}$ RT Reagent Kit (TaKaRa, Kyoto, Japan, Cat. \# RR037B). Primer-BLAST (https://www.ncbi.nlm.nih.gov/tools/primer-blast/index.cgi?LINK_LOC=BlastHome) was used to design gene-specific primers. Wheat house-keeping genes 8 and 34 were used as the reference genes.

Real-time quantitative-PCR (qRT-PCR) was carried out using SYBR ${ }^{\circledR}$ Premix Ex Taq ${ }^{\mathrm{TM}}$ II (Tli RNaseH Plus) (TaKaRa, Kyoto, Japan, Cat. \# RR820A) following the instructions in the manual with a Bio-Rad CFX96 ${ }^{\mathrm{TM}}$ real-time PCR detection system (BioRad, Berkeley, CA, USA). For normalization, three reference genes were used as internal control genes: TaGAPDH, Ta.7894.3.A1_at and Ta.14126.1.S1_at [52,53]. Three biological replicates of tissues were applied for qPCR analyses. The relative gene expression levels were calculated using the $2^{-\Delta \Delta C T}$ method [54], and the primers used in qRT-PCR are listed in Table S3.

\subsection{Subcellular Localization of TaPLATZ}

The subcellular localization of TaPLATZ protein was predicted according to the results of CELLO Version 2.5 (http://cello.life.nctu.edu.tw/) [55], Plant-mPLoc (http://www.csbio.sjtu.edu. cn/bioinf/plant-multi/) [56], WoLF PSORT (https://wolfpsort.hgc.jp/) and SoftBerry (http://linux1. softberry.com/all.htm). Six seed-specifically expressed TaPLATZs were selected to verify the subcellular localization prediction. The similarity of homologous genes make application difficult, the full-length coding sequences of TaPLATZs without stop codon was synthesized by the company, subsequently inserted into pCAMBIA1300 plasmid driven by $35 \mathrm{~S}$ promoter. The resulting vectors were 35S::TaPLATZ26-GFP,35S::TaPLATZ29-GFP, 35S::TaPLATZ32-GFP, 35S::TaPLATZ27-GFP, 35S::TaPLATZ30-GFP, and 35S::TaPLATZ31-GFP, respectively. Agrobacterium-mediated transient transformation of Nicotiana benthamiana plants was conducted to check the subcellular locations of TaPLATZs. Agrobacterium tumefaciens strain GV3101(Weidi, Shanghai, China) carrying expression construct were grown in LB media with kanamycin and Rifampicin antibiotics, diluted in 1:6 and grown for $9 \mathrm{~h}$ at $28^{\circ} \mathrm{C}$, centrifugated for $4000 \mathrm{rpm} 5 \mathrm{~min}$, remove supernatant, pellets were resuspended in infiltration medium (10 $\mathrm{mM} \mathrm{MgCl}_{2}, 10 \mathrm{mM}$ MES-KOH, $\mathrm{PH}$ 5.7, $200 \mu \mathrm{M}$ acetosyringone). The $\mathrm{OD}_{600}$ was adjusted to 0.6-0.9. The resulting culture was infiltrated into 3-week-old $N$. benthamiana leaves using a $1 \mathrm{~mL}$ sterile syringe (no needle). At least three replicates were performed. Subcellular localization was observed after infiltration for 2 days. The GFP fluorescent signal was observed and imaged using a confocal laser scanning microscope (LSM 880, Karl Zeiss, Jena, Germany).

\section{Conclusions}

A total of 62 PLATZ genes were identified in the wheat genome database, and were distributed unevenly on 15 chromosomes. Chromosome 6 contained the most TaPLATZ genes. According to the phylogenetic tree, TaPLATZ genes could be classified into six subfamilies (Group I to VI), Group V and Group VI were without orthologues of other PLATZ genes in other species. Depending on the type of cis-acting elements, TaPLATZ genes may be regulated by a variety of hormones and environmental factors. Gene duplication events analyses suggested that tandem duplication events played a significant role in the expansion of TaPLATZ family. Tissue specificity in different developmental stages can be observed in RNA-seq data and qRT-PCR analysis, suggesting the potential role of TaPLATZs in tissue differentiation and seed development. This study provides valuable information for further understanding of the evolutionary mechanism and functional traits of the PLATZ genes family in wheat.

Supplementary Materials: The following are available online at http://www.mdpi.com/1422-0067/21/23/8934/s1.

Author Contributions: Conceptualization, J.W.; methodology, Y.F.; data analysis, M.C. and X.G.; experimental validation, Y.F. and M.L.; formal analysis, Y.F.; investigation, Y.F.; resources, M.C.; data curation, M.C.; writing—original draft preparation, Y.F.; review and editing, J.W.; visualization, M.C.; supervision, Y.W. and J.W.; 
project administration, J.W.; funding acquisition, Y.W. and J.W. All authors have read and agreed to the published version of the manuscript.

Funding: This research was funded by the National Key Research and Development Program of China (2018YFE0112000; 2017YFD0100900), the National Natural Science Foundation of China (31871609; 91935303), and the Sichuan Science and Technology Support Project (2019YFN0141; 2020YFH0154).

Acknowledgments: We acknowledge the plant research community for sharing the relevant genomic data and making it publicly available.

Conflicts of Interest: The authors declare no conflict of interest. The funders had no role in the design of the study; in the collection, analyses, or interpretation of data; in the writing of the manuscript, or in the decision to publish the results.

\section{Abbreviations}

$\begin{array}{ll}\text { DPA } & \text { Days post-anthesis } \\ \text { HAI } & \text { Hours after imbibition } \\ \text { PLATZ } & \text { plant AT-rich protein and zinc-binding protein } \\ \text { NJ } & \text { Neighbor-Joining } \\ \text { TFs } & \text { Transcription factors }\end{array}$

\section{References}

1. Ning, P.; Liu, C.C.; Kang, J.Q.; Lv, J.Y. Genome-wide analysis of WRKY transcription factors in wheat (Triticum aestivum L.) and differential expression under water deficit condition. Peer] 2017, 5, e3232. [CrossRef] [PubMed]

2. Riechmann, J.L.; Heard, J.; Martin, G.; Reuber, L.; Jiang, C.-Z.; Keddie, J.; Adam, L.; Pineda, O.; Ratcliffe, O.J.; Samaha, R.R.; et al. Arabidopsis transcription factors: Genome-wide comparative analysis among eukaryotes. Science 2000, 290, 2105-2110. [CrossRef] [PubMed]

3. Eulgem, T.; Rushton, P.; Robatzek, S.; Somssich IEulgem, T.; Rushton, P.J.; Robatzek, S.; Somssich, I.E. The WRKY superfamily of plant transcription factors. Trends Plant Sci. 2000, 5, 199-206. [CrossRef]

4. Olsen, A.N.; Ernst, H.A.; Leggio, L.L.; Skriver, K. NAC transcription factors: Structurally distinct, functionally diverse. Trends Plant Sci. 2005, 10, 79-87. [CrossRef] [PubMed]

5. Kizis, D.; Lumbreras, V.; Pagès, M. Role of AP2/EREBP transcription factors in gene regulation during abiotic stress. FEBS Lett. 2001, 498, 187-189. [CrossRef]

6. Yukio, N.; Hirofumi, F.; Takehito, I.; Yukiko, S. A novel class of plant-specific zinc-dependent DNA-binding protein that binds to A/T-rich DNA sequences. Nucleic Acids Res. 2001, 29, 4097-4105. [CrossRef]

7. So, H.A.; Choi, S.J.; Chung, E.; Lee, J.H. Molecular characterization of stress-inducible PLATZ gene from soybean (Glycine max L.). Plant Omics 2015, 8, 479-484.

8. Li, Q.; Wang, J.; Ye, J.; Zheng, X.; Xiang, X.; Li, C.; Fu, M.; Wang, Q.; Zhang, Z.; Wu, Y. The Maize Imprinted Gene Floury3 Encodes a PLATZ Protein Required for tRNA and 5S rRNA Transcription through Interaction with RNA Polymerase III. Plant Cell 2017, 29, 2661-2675. [CrossRef]

9. Kim, J.H.; Kim, J.; Jun, S.E.; Park, S.; Timilsina, R.; Kwon, D.S.; Kim, Y.; Park, S.-J.; Hwang, J.Y.; Nam, H.G.; et al. ORESARA15, a PLATZ transcription factor, mediates leaf growth and senescence in Arabidopsis. New Phytol. 2018, 220, 609-623. [CrossRef]

10. Wang, A.; Hou, Q.; Si, L.; Huang, X.; Luo, J.; Lu, D.; Zhu, J.; Shangguan, Y.; Miao, J.; Xie, Y.; et al. The PLATZ Transcription Factor GL6 Affects Grain Length and Number in Rice. Plant Physiol. 2019, 180, 2077-2090. [CrossRef]

11. Zhou, S.R.; Xue, H.W. The rice PLATZ protein SHORT GRAIN6 determines grain size by regulating spikelet hull cell division. J. Integr. Plant Biol. 2019, 62, 847-864. [CrossRef] [PubMed]

12. Wang, J.; Ji, C.; Li, Q.; Zhou, Y.; Wu, Y. Genome-wide analysis of the plant-specific PLATZ proteins in maize and identification of their general role in interaction with RNA polymerase III complex. BMC Plant Biol. 2018, 18, 221. [CrossRef] [PubMed]

13. Heun, M.; SchaferPregl, R.; Klawan, D.; Castagna, R.; Accerbi, M.; Borghi, B.; Salamini, F. Site of einkorn wheat domestication identified by DNA fingerprinting. Science 1997, 278, 1312-1314. [CrossRef] 
14. Marcussen, T.; Sandve, S.R.; Heier, L.; Spannagl, M.; Pfeifer, M.; Jakobsen, K.S.; Wulff, B.B.H.; Steuernagel, B.; Mayer, K.F.X.; Olsen, O.A. Ancient hybridizations among the ancestral genomes of bread wheat. Science 2014, 345, 286-292. [CrossRef] [PubMed]

15. Matsuoka, Y.; Nasuda, S. Durum wheat as a candidate for the unknown female progenitor of bread wheat: An empirical study with a highly fertile F1 hybrid with Aegilops tauschii Coss. Theor. Appl. Genet. 2004, 109, 1710-1717. [CrossRef]

16. Appels, R.; Eversole, K.; Stein, N.; Feuillet, C.; Keller, B.; Rogers, J.; Pozniak, C.J.; Choulet, F.; Distelfeld, A.; Poland, J.; et al. Shifting the limits in wheat research and breeding using a fully annotated reference genome. Science 2018, 361, 661-674. [CrossRef]

17. Avni, R.; Nave, M.; Barad, O.; Baruch, K.; Twardziok, S.O.; Gundlach, H.; Hale, I.; Mascher, M.; Spannagl, M.; Wiebe, K.; et al. Wild emmer genome architecture and diversity elucidate wheat evolution and domestication. Science 2017, 357, 93-97. [CrossRef]

18. Koenig, D.; Jimenez-Gomez, J.M.; Kimura, S.; Fulop, D.; Chitwood, D.H.; Headland, L.R.; Kumar, R.; Covington, M.F.; Devisetty, U.K.; Tat, A.V.; et al. Comparative transcriptomics reveals patterns of selection in domesticated and wild tomato. Proc. Natl. Acad. Sci. USA 2013, 110, E2655-E2662. [CrossRef]

19. Huang, X.; Kurata, N.; Wei, X.; Wang, Z.-X.; Wang, A.; Zhao, Q.; Zhao, Y.; Liu, K.; Lu, H.; Li, W.; et al. A map of rice genome variation reveals the origin of cultivated rice. Nature 2012, 490, 497-501. [CrossRef]

20. Uauy, C.; Wulff, B.B.H.; Dubcovsky, J. Combining Traditional Mutagenesis with New High-Throughput Sequencing and Genome Editing to Reveal Hidden Variation in Polyploid Wheat. Annu. Rev. Genet. 2017, 51, 435-454. [CrossRef]

21. Conte, M.G.; Gaillard, S.; Lanau, N.; Rouard, M.; Perin, C. GreenPhylDB: A database for plant comparative genomics. Nucleic Acids Res. 2007, 36, D991-D998. [CrossRef] [PubMed]

22. Schilling, S.; Kennedy, A.; Pan, S.; Jermiin, L.; Melzer, R. Genome-wide analysis of MIKC-type MADS-box genes in wheat: Pervasive duplications may have facilitated adaptation to different environmental conditions. New Phytol. 2019, 225, 511-529. [CrossRef] [PubMed]

23. Hurles, M. Gene duplication: The genomic trade in spare parts. PLoS Biol. 2004, 2, 900-904. [CrossRef] [PubMed]

24. Hernandez-Garcia, C.M.; Finer, J.J. Identification and validation of promoters and cis-acting regulatory elements. Plant Sci. 2014, 109, 217-218. [CrossRef] [PubMed]

25. Reidt, W.; Wohlfarth, T.; Ellerström, M.; Czihal, A.; Bäumlein, H. Gene regulation during late embryogenesis: The RY motif of maturation-specific gene promoters is a direct target of the FUS3 gene product. Plant J. 2000, 21, 401-408. [CrossRef]

26. Otto, S.P.; Whitton, J. Polyploid incidence and evolution. Annu. Rev. Genet. 2000, 34, 401-437. [CrossRef]

27. Blanc, G. A Recent Polyploidy Superimposed on Older Large-Scale Duplications in the Arabidopsis Genome. Genome Res. 2003, 13, 137-144. [CrossRef]

28. Panchy, N.; Lehti-Shiu, M.D.; Shiu, S.H. Evolution of Gene Duplication in Plants. Plant Physiol. 2016, 171, 2294-2316. [CrossRef]

29. Moore, R.C.; Purugganan, M.D. The early stages of duplicate gene evolution. Proc. Natl. Acad. Sci. USA 2003, 100, 15682-15687. [CrossRef]

30. Feldman, M.; Levy, A.A. Genome Evolution Due to Allopolyploidization in Wheat. Genetics 2012, 192, 763-774. [CrossRef]

31. Liu, Y.; Liu, N.N.; Deng, X.; Liu, D.M.; Li, M.F.; Cui, D.D.; Hu, Y.K.; Yan, Y.M. Genome-wide analysis of wheat DNA-binding with one finger (Dof) transcription factor genes: Evolutionary characteristics and diverse abiotic stress responses. BMC Genom. 2020, 21, 1-18. [CrossRef]

32. Rahman, S.; Islam, S.; Yu, Z.; She, M.; Nevo, E.; Ma, W. Current Progress in Understanding and Recovering the Wheat Genes Lost in Evolution and Domestication. Int. J. Mol. Sci. 2020, 21, 5836. [CrossRef] [PubMed]

33. Edger, P.P.; Pires, J.C. Gene and genome duplications: The impact of dosage-sensitivity on the fate of nuclear genes. Chromosome Res. 2009, 17, 699-717. [CrossRef] [PubMed]

34. Kong, H.; Landherr, L.L.; Frohlich, M.W.; Leebens-Mack, J.; Ma, H.; DePamphilis, C.W. Patterns of gene duplication in the plant SKP1 gene family in angiosperms: Evidence for multiple mechanisms of rapid gene birth. Plant J. 2007, 50, 873-885. [CrossRef] [PubMed] 
35. Adams, K.L.; Wendel, J.F. Polyploidy and genome evolution in plants. Curr. Opin. Plant Biol. 2005, 8, $135-141$. [CrossRef]

36. Cannon, S.B.; Mitra, A.; Baumgarten, A.; Young, N.D.; May, G. The roles of segmental and tandem gene duplication in the evolution of large gene families in Arabidopsis thaliana. BMC Plant Biol. 2004, 4, 10. [CrossRef]

37. Force, A.; Lynch, M.; Pickett, F.B.; Amores, A.; Yan, Y.L.; Postlethwait, J. Preservation of duplicate genes by complementary, degenerative mutations. Genetics 1999, 151, 1531-1545.

38. Lynch, M.; Force, A. The probability of duplicate gene preservation by subfunctionalization. Genetics 2000, 154, 459-473.

39. Kiełbowicz-Matuk, A. Involvement of plant $\mathrm{C}_{2} \mathrm{H}_{2}$-type zinc finger transcription factors in stress responses. Plant Sci. Int. J. Exp. Plant Biol. 2012, 185-186, 78-85. [CrossRef]

40. González-Morales, S.I.; Chávez-Montes, R.A.; Hayano-Kanashiro, C.; Alejo-Jacuinde, G.; Rico-Cambron, T.Y.; de Folter, S.; Herrera-Estrella, L. Regulatory network analysis reveals novel regulators of seed desiccation tolerance in Arabidopsis thaliana. Proc. Natl. Acad. Sci. USA 2016, 113, E5232. [CrossRef]

41. Zhang, S.; Yang, R.; Huo, Y.; Liu, S.; Yang, G.; Huang, J.; Zheng, C.; Wu, C. Expression of cotton PLATZ1 in transgenic Arabidopsis reduces sensitivity to osmotic and salt stress for germination and seedling establishment associated with modification of the abscisic acid, gibberellin, and ethylene signalling pathways. BMC Plant Biol. 2018, 18, 1-11. [CrossRef] [PubMed]

42. Rinerson, C.I.; Rabara, R.C.; Tripathi, P.; Shen, Q.J.; Rushton, P.J. The evolution of WRKY transcription factors. BMC Plant Biol. 2015, 15, 1-18. [CrossRef] [PubMed]

43. Bailey, T.L.; Boden, M.; Buske, F.A.; Frith, M.; Grant, C.E.; Clementi, L.; Ren, J.; Li, W.W.; Noble, W.S. MEME SUITE: Tools for motif discovery and searching. Nucleic Acids Res. 2009, 37, W202-W208. [CrossRef] [PubMed]

44. Chen, C.; Chen, H.; Zhang, Y.; Thomas, H.R.; Frank, M.H.; He, Y.; Xia, R. TBtools: An Integrative Toolkit Developed for Interactive Analyses of Big Biological Data. Mol. Plant 2020, 13, 1194-1202. [CrossRef] [PubMed]

45. Magali, L. PlantCARE, a database of plant cis-acting regulatory elements and a portal to tools for in silico analysis of promoter sequences. Nucleic Acids Res. 2002, 1, 325-327. [CrossRef]

46. Tamura, K.; Stecher, G.; Peterson, D.; Filipski, A.; Kumar, S. MEGA6: Molecular Evolutionary Genetics Analysis Version 6.0. Mol. Biol. Evol. 2013, 30, 2725-2729. [CrossRef]

47. Krzywinski, M.; Schein, J.; Birol, I.; Connors, J.; Gascoyne, R.; Horsman, D.; Jones, S.J.; Marra, M.A. Circos: An information aesthetic for comparative genomics. Genome Res. 2009, 19, 1639-1645. [CrossRef]

48. Wang, M.; Yue, H.; Feng, K.; Deng, P.; Song, W.; Nie, X. Genome-wide identification, phylogeny and expressional profiles of mitogen activated protein kinase kinase kinase (MAPKKK) gene family in bread wheat (Triticum aestivum L.). BMC Genom. 2016, 17, 668. [CrossRef]

49. Zhao, Y.; Ma, R.; Xu, D.; Bi, H.; Xia, Z.; Peng, H. Genome-Wide Identification and Analysis of the AP2 Transcription Factor Gene Family in Wheat (Triticum aestivum L.). Front. Plant Sci. 2019, 10, 1286. [CrossRef]

50. Holub, E.B. The arms race is ancient history in Arabidopsis, the wildflower. Nat. Rev. Genet. 2001, 2, 516-527. [CrossRef]

51. Bi, C.; Xu, Y.; Ye, Q.; Yin, T.; Ye, N. Genome-wide identification and characterization of WRKY gene family inSalix suchowensis. PeerJ 2016, 4, e2437. [CrossRef] [PubMed]

52. Jarosová, J.; Kundu, J.K. Validation of reference genes as internal control for studying viral infections in cereals by quantitative real-time RT-PCR. BMC Plant Biol. 2010, 10, 146. [CrossRef] [PubMed]

53. Long, X.-Y.; Wang, J.-R.; Ouellet, T.; Rocheleau, H.; Wei, Y.-M.; Pu, Z.-E.; Jiang, Q.-T.; Lan, X.-J.; Zheng, Y.-L. Genome-wide identification and evaluation of novel internal control genes for Q-PCR based transcript normalization in wheat. Plant Mol. Biol. 2010, 74, 307-311. [CrossRef]

54. Livak, K.J.; Schmittgen, T.D. Analysis of Relative Gene Expression Data Using Real-Time Quantitative PCR and the $2^{-\Delta \Delta C T}$ Method. Methods 2001, 25, 402-408. [CrossRef] 
55. Yu, C.-S.; Chen, Y.-C.; Lu, C.-H.; Hwang, J.-K. Prediction of protein subcellular localization. Proteins Struct. Funct. Bioinform. 2006, 64, 643-651. [CrossRef]

56. Chou, K.-C.; Shen, H.-B. Plant-mPLoc: A Top-Down Strategy to Augment the Power for Predicting Plant Protein Subcellular Localization. PLoS ONE 2010, 5, e11335. [CrossRef]

Publisher's Note: MDPI stays neutral with regard to jurisdictional claims in published maps and institutional affiliations.

(C) 2020 by the authors. Licensee MDPI, Basel, Switzerland. This article is an open access article distributed under the terms and conditions of the Creative Commons Attribution (CC BY) license (http://creativecommons.org/licenses/by/4.0/). 